M. Mustaţă and K. Yoshida

Nagoya Math. J.

Vol. 193 (2009), 111-128

\title{
TEST IDEALS VS. MULTIPLIER IDEALS
}

\author{
MIRCEA MUSTAŢĂ AND KEN-ICHI YOSHIDA
}

\begin{abstract}
The generalized test ideals introduced in [HY] are related to multiplier ideals via reduction to characteristic $p$. In addition, they satisfy many of the subtle properties of the multiplier ideals, which in characteristic zero follow via vanishing theorems. In this note we give several examples to emphasize the different behavior of test ideals and multiplier ideals. Our main result is that every ideal in an $F$-finite regular local ring can be written as a generalized test ideal. We also prove the semicontinuity of $F$-pure thresholds (though the analogue of the Generic Restriction Theorem for multiplier ideals does not hold).
\end{abstract}

\section{$\S 1$. Introduction}

In recent years the multiplier ideals and the log canonical threshold have played an important role in higher dimensional birational geometry (see e.g. [Laz]). These are invariants of singularities in characteristic zero, that can be defined in terms of $\log$ resolutions of singularities. Suppose for simplicity that $X$ is a smooth variety over a field of characteristic zero, and that $\mathfrak{a} \subseteq \mathcal{O}_{X}$ is a coherent sheaf of ideals. The multiplier ideal associated to the pair $(X, \mathfrak{a})$ and to a non-negative real number $t$ is denoted by $\mathcal{J}\left(\mathfrak{a}^{t}\right)$. If $t_{1}<t_{2}$, then $\mathcal{J}\left(\mathfrak{a}^{t_{2}}\right) \subseteq \mathcal{J}\left(\mathfrak{a}^{t_{1}}\right)$, and $\mathcal{J}\left(\mathfrak{a}^{t}\right)=\mathcal{O}_{X}$ for $0<t \ll 1$. The smallest $t$ such that $\mathcal{J}\left(\mathfrak{a}^{t}\right) \neq \mathcal{O}_{X}$ is the log canonical threshold lct $(\mathfrak{a})$.

On the other hand, in positive characteristic one can define invariants using the Frobenius morphism. Specifically, Hara and the second author introduced in $[\mathrm{HY}]$ a notion of tight closure for pairs, and corresponding (generalized) test ideals $\tau\left(\mathfrak{a}^{t}\right)$. Suppose that we have a pair $(X, \mathfrak{a})$ and $t \in \mathbb{R}_{+}$, where $X$ is a smooth variety over a field of characteristic zero. If

Received July 27, 2007.

Revised April 23, 2008.

Accepted May 26, 2008 .

2000 Mathematics Subject Classification: Primary 13A35; Secondary 14B05.

The first author was partially supported by NSF grant DMS 0500127 and by a Packard Fellowship. The second author was partially supported by Grant-in-Aid for Scientific Research, 19340005 from JSPS. 
we denote by $\mathfrak{b}_{p}$ the reduction $\bmod p$ of the ideal $\mathfrak{b}$, it was proved in $[\mathrm{HY}]$ that

$$
\mathcal{J}\left(\mathfrak{a}^{t}\right)_{p}=\tau\left(\mathfrak{a}_{p}^{t}\right)
$$

for all primes $p \gg 0$ (depending on $t$ ).

In the same vein, Takagi and Watanabe defined in positive characteristic [TW] the $F$-pure threshold $\operatorname{fpt}(\mathfrak{a})$. When the ambient variety is nonsingular and $F$-finite (that is, the Frobenius morphism $F: X \rightarrow X$ is finite), this can be described as the smallest $t$ such that $\tau\left(\mathfrak{a}^{t}\right) \neq \mathcal{O}_{X}$. The formula (1) can then be reinterpreted as saying that

$$
\lim _{p \rightarrow \infty} \operatorname{fpt}\left(\mathfrak{a}_{p}\right)=\operatorname{lct}(\mathfrak{a})
$$

The above shows the close connection between multiplier and test ideals. In fact, more is true. Multiplier ideals satisfy several subtle properties, such as the Restriction Theorem, the Subadditivity and the Summation Theorems, and Skoda's Theorem (see [Laz]). One common feature of these results is that they all rely on applications of vanishing theorems. As it was pointed out in $[\mathrm{HY}],[\mathrm{HT}]$ and $[\mathrm{Ta}]$, all these results have similar statements for test ideals, with substantially easier proofs.

On the other hand, multiplier ideals enjoy several other properties, that follow simply from the description in terms of resolutions of singularities. In this note we concentrate on these properties, and show that essentially all these fail for test ideals.

Our basic ingredient is the description of test ideals from [BMS1], which holds when the ambient variety is nonsingular and $F$-finite. Therefore we will always make this assumption. Our main result is a positive one: under mild assumptions, every ideal is a test ideal.

THEOREM 1.1. Suppose that $R$ is a ring of characteristic $p>0$, such that $R$ is a finitely generated free module over $R^{p}$. For every ideal $I$ in $R$, there is $f \in R$ and $c>0$ such that $I=\tau\left(f^{c}\right)$.

Note that the theorem applies when $R$ is a local regular $F$-finite ring, or when $R=k\left[x_{1}, \ldots, x_{n}\right]$, where $\left[k: k^{p}\right]<\infty$. As we will see, both $f$ and $c$ in the theorem can be explicitly determined. Moreover, if $I$ is $\mathfrak{m}$-primary, for some maximal ideal $\mathfrak{m}$, then we show that we may write also $I=\tau\left(\mathfrak{a}^{c^{\prime}}\right)$ for some $\mathfrak{m}$-primary ideal $\mathfrak{a}$ and some $c^{\prime}>0$. 
Note that Theorem 1.1 contrasts with the situation for multiplier ideals. In that case, as an immediate consequence of the definition one shows that every multiplier ideal is integrally closed. Moreover, as it was recently shown in $[\mathrm{LL}]$, there are more subtle conditions involving the local syzygies, that are satisfied by all multiplier ideals.

In [ELSV] one shows that whenever one writes an ideal $I$ as a multiplier ideal, then one can prove an effective uniform Artin-Rees theorem for $I$. The main ingredient in that proof is a basic property of multiplier ideals that follows from the definition via resolutions. As we show in Example 4.5 below, this property fails in the case of test ideals, and therefore it seems that Theorem 1.1 does not have similar consequences in the direction of uniform Artin-Rees statements.

We give several examples to illustrate that basic properties of multiplier ideals, which easily follow from the definition via log resolutions, can fail in the case of test ideals:

i) We show that it can happen that for a (principal) ideal $\mathfrak{a}$, we can have the ideal $\tau\left(\mathfrak{a}^{c}\right)$ non-radical, where $c=\operatorname{fpt}(\mathfrak{a})$ (see Example 4.3).

ii) We give an example of a (principal) ideal $\mathfrak{a}$ with $c=\operatorname{fpt}(\mathfrak{a})$ such that $\tau\left(\mathfrak{a}^{c}\right)$ is $\mathfrak{m}$-primary for a maximal ideal $\mathfrak{m}$, but such that $\operatorname{fpt}(\mathfrak{a})<$ $\operatorname{fpt}\left(\mathfrak{a}+\mathfrak{m}^{\ell}\right)$ for all $\ell \gg 0$ (see Example 4.3).

iii) We show that the analogue of the Generic Restriction Theorem for multiplier ideals can fail in the case of test ideals (see Example 4.7). However, we will prove that the $F$-pure thresholds satisfy the same semicontinuity property as the log canonical thresholds.

The paper is structured as follows. In Section 2 we review the definitions of multiplier and generalized test ideals, and some basic properties. In particular, we recall the description of test ideals in the case of a regular $F$-finite ring from [BMS1], which we will systematically use. In Section 3 we prove Theorem 1.1 above. The next section is devoted to various examples, including the ones mentioned above, while in the last section we prove the semicontinuity result for $F$-pure thresholds.

\section{§2. Preliminaries}

We first recall the definition of multiplier ideals (for details see [Laz, §9]). For a real number $u$, we denote by $\lceil u\rceil$ the smallest integer $\geq u$. Similarly, $\lfloor u\rfloor$ is the largest integer $\leq u$. This notation is extended to divisors with real coefficients, in which case we apply it to each coefficient. 
Let $X$ be a $\mathbb{Q}$-Gorenstein normal variety over a field of characteristic zero, $Y \subsetneq X$ a proper closed subscheme defined by an ideal sheaf $\mathfrak{a} \subseteq \mathcal{O}_{X}$, and $t \geq 0$ a real number. Suppose that $\pi: \widetilde{X} \rightarrow X$ is a $\log$ resolution of the pair $(X, Y)$ such that $\mathfrak{a} \mathcal{O}_{\widetilde{X}}=\mathcal{O}_{\widetilde{X}}(-F)$, and let $K_{\widetilde{X} / X}$ denote the discrepancy divisor. Then the multiplier ideal $\mathcal{J}\left(\mathfrak{a}^{t}\right)$ is defined by

$$
\mathcal{J}\left(\mathfrak{a}^{t}\right)=\pi_{*} \mathcal{O}_{\widetilde{X}}\left(\left\lceil K_{\widetilde{X} / X}-t F\right\rceil\right) \subseteq \mathcal{O}_{X}
$$

This is an ideal of $\mathcal{O}_{X}$ that does not depend on the choice of the log resolution.

One says that $X$ has $\log$ terminal singularities at $x \in X$ if $x$ does not lie in the support of $\mathcal{J}\left(\mathfrak{a}^{t}\right)$ for $0<t \ll 1$. In this case one defines the $\log$ canonical threshold of $\mathfrak{a}$ at $x$, denoted by $\operatorname{lct}_{x}(\mathfrak{a})$, to be

$$
\operatorname{lct}_{x}(\mathfrak{a})=\sup \left\{s \in \mathbb{R}_{\geq 0} \mid x \text { is not in the support of } \mathcal{J}\left(\mathfrak{a}^{s}\right)\right\}
$$

For the purpose of this paper, it is enough to restrict ourselves to the case when the variety $X$ is nonsingular (hence, in particular, $X$ has log terminal singularities at every point). It is easy to see starting from definition that $\mathcal{J}\left(\mathfrak{a}^{t_{1}}\right) \subseteq \mathcal{J}\left(\mathfrak{a}^{t_{2}}\right)$ if $t_{1}>t_{2}$. Moreover, given any $t \geq 0$, there is a positive $\varepsilon$ such that $\mathcal{J}\left(\mathfrak{a}^{t}\right)=\mathcal{J}\left(\mathfrak{a}^{t+\varepsilon}\right)$. Following [ELSV], we call $\lambda>0$ a jumping number of $\mathfrak{a}$ if $\mathcal{J}\left(\mathfrak{a}^{\lambda}\right) \neq \mathcal{J}\left(\mathfrak{a}^{t}\right)$ for every $t<\lambda$. With the notation in the definition of multiplier ideals, it follows easily that if we write $F=\sum_{i} a_{i} E_{i}$, then for every jumping number $\lambda$ of $\mathfrak{a}$, there is $i$ such that $a_{i} \lambda$ is an integer. In particular, the jumping numbers are rational and they form a discrete set.

The smallest jumping number of $\mathfrak{a}$ is the log canonical threshold $\operatorname{lct}(\mathfrak{a})$. It is clear that we can define local versions of the jumping numbers at every $x \in X$. In this case, the smallest jumping number is precisely $\operatorname{lct}_{x}(\mathfrak{a})$. In fact, it is easy to see that $\operatorname{lct}(\mathfrak{a})=\min _{x \in X} \operatorname{lct}_{x}(\mathfrak{a})$.

We now turn to the positive characteristic setting. Let $R$ be a Noetherian ring containing a field of characteristic $p>0$. The ring $R$ is called $F$-finite if $R$ is a finitely generated module over its subring $R^{p}=\left\{a^{p} \in R\right.$ : $a \in R\}$. If $J$ is an ideal in $R$, then $J^{\left[p^{e}\right]}$ denotes the ideal $\left(u^{p^{e}}: u \in J\right)$. We recall first the notion of generalized test ideals, introduced by Hara and the second author in $[\mathrm{HY}]$. We denote by $R^{\circ}$ the complement of all minimal prime ideals of $R$. 
Definition 2.1. Let $\mathfrak{a}$ be an ideal such that $\mathfrak{a} \cap R^{\circ} \neq \emptyset$. Let $t \geq 0$ be a real number. For any ideal $I$ of $R$, the $\mathfrak{a}^{t}$-tight closure of $I$, denoted by $I^{* \mathfrak{a}^{t}}$, is defined to be the ideal of $R$ consisting of all elements $z \in R$ for which there exists $c \in R^{\circ}$ such that

$$
c z^{q} \mathfrak{a}^{[t q\rceil} \subseteq I^{[q]}
$$

for all large $q=p^{e}$.

Assume that $R$ is excellent and reduced. Given a real number $t \geq 0$, one defines the generalized test ideal $\tau\left(\mathfrak{a}^{t}\right)$ by

$$
\tau\left(\mathfrak{a}^{t}\right)=\bigcap_{I \subseteq R} I: I^{* \mathfrak{a}^{t}}
$$

where $I$ runs through all ideals of $R$. In the case of a principal ideal $\mathfrak{a}=(f)$, we simply write $\tau\left(f^{t}\right)$.

Blickle, Smith and the first author gave in [BMS1] a different description of generalized test ideals in the case of an $F$-finite regular ring $R$. We briefly recall this description here, in the special case when $R$ is free and finitely generated over $R^{p}$. Note that this condition holds, for example, when $R$ is an $F$-finite regular local ring, or when $R=k\left[x_{1}, \ldots, x_{n}\right]$ and $\left[k: k^{p}\right]<\infty$.

It follows from our assumption that for every $p^{e}$, with $e \geq 1, R$ is free over $R^{p^{e}}=\left\{a^{p^{e}}: a \in R\right\}$. For every such $e$, let us fix a basis $u_{1}, \ldots, u_{N}$ of $R$ over $R^{p^{e}}$. Given any ideal $\mathfrak{b}$ of $R$, we choose generators $h_{1}, \ldots, h_{s}$ of $\mathfrak{b}$. If we write for every $i$

$$
h_{i}=\sum_{j=1}^{N} a_{i j}^{p^{e}} u_{j},
$$

with $a_{i j} \in R$, then we put

$$
\mathfrak{b}^{\left[1 / p^{e}\right]}=\left(a_{i j}: 1 \leq i \leq s, 1 \leq j \leq N\right) .
$$

In fact, $\mathfrak{b}^{\left[1 / p^{e}\right]}$ is the unique smallest ideal $J$ (with respect to inclusion) such that $\mathfrak{b} \subseteq J^{\left[p^{e}\right]}$. In particular, $\mathfrak{b}^{\left[1 / p^{e}\right]}$ does not depend on the choice of generators for $\mathfrak{b}$, or on the choice of basis for $R$ over $R^{p^{e}}$.

Suppose now that $\mathfrak{a}$ is an ideal in $R$ and that $t$ is a positive real number. For every $e \geq 1$ we have the inclusion

$$
\left(\mathfrak{a}^{\left[t p^{e}\right\rceil}\right)^{\left[1 / p^{e}\right]} \subseteq\left(\mathfrak{a}^{\left\lceil t p^{e+1}\right\rceil}\right)^{\left[1 / p^{e+1}\right]}
$$


Since $R$ is Noetherian, these ideals stabilize for $e \gg 0$, and the limit was taken as definition for $\tau\left(\mathfrak{a}^{t}\right)$ in loc. cit, the equivalence with the definition from [HY] being proved in ibid., Proposition 2.22.

We now recall the definition of $F$-jumping exponents, that is analogous to that of jumping numbers for multiplier ideals. We assume that $R$ is a regular $F$-finite ring. Note that if $t<t^{\prime}$, then $\tau\left(\mathfrak{a}^{t}\right) \supseteq \tau\left(\mathfrak{a}^{t^{\prime}}\right)$. Moreover, for every $t$ there exists $\varepsilon>0$ such that $\tau\left(\mathfrak{a}^{t}\right)=\tau\left(\mathfrak{a}^{t^{\prime}}\right)$ for every $t^{\prime} \in[t, t+\varepsilon)$.

Definition 2.2. A positive real number $\lambda$ is called an F-jumping exponent of $\mathfrak{a}$ if $\tau\left(\mathfrak{a}^{\lambda}\right) \neq \tau\left(\mathfrak{a}^{t}\right)$ for every $t<\lambda$. It is convenient to make also the convention that 0 is an $F$-jumping exponent.

The smallest positive $F$-jumping exponent of $\mathfrak{a}$ is the $F$-pure threshold $\operatorname{fpt}(\mathfrak{a})$. This notion was introduced in a more general setting by Takagi and Watanabe in $[\mathrm{TW}]$, as an analogue of the log canonical threshold.

When $(R, \mathfrak{m})$ is an $F$-finite regular local ring, the $F$-pure threshold has the following alternative description (see [BMS1] or [MTW]). Given an ideal $\mathfrak{a} \subseteq \mathfrak{m}$ and $e \geq 1$, we denote by $\nu(e)$ the largest integer $r$ such that $\mathfrak{a}^{r} \nsubseteq \mathfrak{m}^{\left[p^{e}\right]}$ (we put $\nu(e)=0$ if there is no such $r$ ). We then have

$$
\operatorname{fpt}(\mathfrak{a})=\sup _{e} \frac{\nu(e)}{p^{e}} .
$$

It follows that given a nonnegative integer $c$, we have $\operatorname{fpt}(\mathfrak{a}) \leq c$ if and only if $\mathfrak{a}^{\left\lfloor c p^{e}\right\rfloor+1} \subseteq \mathfrak{m}^{\left[p^{e}\right]}$ for every $e$.

Rationality and discreteness of $F$-jumping exponents is more subtle in positive characteristic. Both properties have been proved in [BMS1] for an arbitrary ideal in a regular ring that is essentially of finite type over an $F$-finite field, and for a principal ideal in any $F$-finite regular ring in [BMS2].

We will be especially interested in the case when $\mathfrak{a}=(f)$ is a principal ideal in an F-finite regular ring. In this case, Skoda's Theorem (see Theorem 4.1 in $[\mathrm{HT}]$ or Proposition 2.25 in [BMS1]) implies that for every $t \geq 1$ we have $\tau\left(f^{t}\right)=f \cdot \tau\left(f^{t-1}\right)$. Therefore the set of $F$-jumping exponents of $f$ is periodic with period one, hence it is enough to describe the $F$-jumping exponents in the interval $(0,1]$. As we have mentioned, this is a finite set. 


\section{§3. Any ideal in an $\boldsymbol{F}$-finite regular local ring is a test ideal}

Throughout this section we assume that $R$ is a regular, $F$-finite ring. By a theorem of Kunz $[\mathrm{Ku}]$, this is equivalent with $R$ being finitely generated and projective over $R^{p}$. We will assume that moreover, $R$ is free over $R^{p}$. This holds, for example, if $R$ is also local, or if $R=k\left[x_{1}, \ldots, x_{n}\right]$, where $\left[k: k^{p}\right]<\infty$. The following is the main result of this section.

THEOREM 3.1. Let $R$ be a regular ring of characteristic $p>0$, such that $R$ is a finitely generated, free module over $R^{p}$.

1) For every ideal $I$ in $R$, there are $f \in R$ and $c>0$ such that $I=\tau\left(f^{c}\right)$.

2) Moreover, if $\mathfrak{m}$ is a maximal ideal in $R$, and if $I$ is $\mathfrak{m}$-primary, then we can find an $\mathfrak{m}$-primary ideal $\mathfrak{b}$ and $c^{\prime}>0$ such that $I=\tau\left(\mathfrak{b}^{c^{\prime}}\right)$.

Suppose that $R$ satisfies the hypothesis of the theorem, and let $N=$ $\operatorname{rk}_{R^{p}}(R)$. It is clear that $N=1$ if and only if $\operatorname{dim}(R)=0$, in which case Theorem 3.1 is trivial. We will henceforth assume $N>1$. Note that if $e \geq 1$, then $R$ is free over $R^{e}$ of rank $N^{e}$.

The first assertion in Theorem 3.1 follows from the more precise statement below.

Proposition 3.2. Let $R$ be a ring of characteristic $p>0$ that is free and finitely generated over $R^{p}$, with $\operatorname{rk}_{R^{p}}(R)=N$. Let $I=\left(z_{1}, \ldots, z_{\mu}\right)$ be an ideal of $R$, and fix $e_{0} \geq 1$ such that $N^{e_{0}} \geq \mu$. If $g_{1}, \ldots, g_{N^{e_{0}}}$ is a basis of $R$ over $R^{p^{e_{0}}}$, and if we put

$$
f=\sum_{i=1}^{\mu} z_{i}^{p^{e_{0}}} g_{i} \in R, \quad c=\frac{1}{p^{e_{0}}} \in \mathbb{Q},
$$

then

$$
\tau\left(f^{c}\right)=I
$$

Proof. We use the description of $\tau\left(f^{c}\right)$ from [BMS1]. If $e \geq e_{0}$, then we have a basis of $R$ over $R^{p^{e}}$ given by

$$
\left\{g_{i_{1}} g_{i_{2}}^{p} \cdots g_{i_{e-e_{0}+1}^{p^{e-e_{0}}}} \mid 1 \leq i_{1}, \ldots, i_{e-e_{0}+1} \leq N\right\} .
$$

Since we can write $f^{p^{e-e_{0}}}=\sum_{i=1}^{\mu} z_{i}^{p^{e}} g_{i}^{p^{e-e_{0}}}$, it follows that

$$
\left(f^{\left\lceil c p^{e}\right\rceil}\right)^{\left[1 / p^{e}\right]}=\left(f^{p^{e-e_{0}}}\right)^{\left[1 / p^{e}\right]}=\left(z_{1}, \ldots, z_{\mu}\right)=I .
$$

Since this is true for every $e \geq e_{0}$, we deduce $\tau\left(f^{c}\right)=I$. 
We turn now to the second assertion in Theorem 3.1 (this answers positively a question raised by Kei-ichi Watanabe). The assertion is a consequence of 1), together with the more general statement below. Recall that by Corollary 2.16 in [BMS1], for every $f$ and every $c$ there is $\varepsilon>0$ such that $\tau\left(f^{c}\right)=\tau\left(f^{c+\varepsilon}\right)$.

Proposition 3.3. Let $R$ be a regular $F$-finite ring, and $\mathfrak{m}$ a maximal ideal in $R$. Suppose that $f \in R$ and $c>0$ are such that $I:=\tau\left(f^{c}\right)$ is $\mathfrak{m}$ primary. If we fix $\varepsilon>0$ such that $I=\tau\left(f^{c+\varepsilon}\right)$, and if $r$ is such that $\mathfrak{m}^{r} \subseteq I$, then for every positive integer $\ell$ with $\ell \varepsilon \geq r+\operatorname{codim}(\mathfrak{m})-1$, we have

$$
I=\tau\left(\left(f R+\mathfrak{m}^{\ell}\right)^{c+\varepsilon}\right) .
$$

Proof. We put $\mathfrak{a}_{\ell}=f R+\mathfrak{m}^{\ell}$. Note that we clearly have $I=\tau\left(f^{c+\varepsilon}\right) \subseteq$ $\tau\left(\mathfrak{a}_{\ell}^{c+\varepsilon}\right)$.

On the other hand, by Takagi's Summation Theorem (see Theorem 3.1 in [Ta]), we have

$$
\tau\left(\mathfrak{a}_{\ell}^{c+\varepsilon}\right) \subseteq \sum_{\lambda+\nu=c+\varepsilon} \tau\left(f^{\lambda}\right) \cdot \tau\left(\mathfrak{m}^{\ell \nu}\right) \subseteq \tau\left(f^{c}\right)+\tau\left(\mathfrak{m}^{\ell \varepsilon}\right)
$$

For the second inclusion we used the fact that if $\lambda \geq c$, then $\tau\left(f^{\lambda}\right) \subseteq \tau\left(f^{c}\right)$, and otherwise we have $\nu \geq \varepsilon$, hence $\tau\left(\mathfrak{m}^{\ell \nu}\right) \subseteq \tau\left(\mathfrak{m}^{\ell \varepsilon}\right)$.

Since $\ell \varepsilon \geq r+d-1$, where $d=\operatorname{codim}(\mathfrak{m})$, and since $\tau\left(\mathfrak{m}^{\alpha}\right)=\mathfrak{m}^{\lfloor\alpha\rfloor-d+1}$ for every $\alpha \geq d-1$, it follows that

$$
\tau\left(\mathfrak{m}^{\ell \varepsilon}\right) \subseteq \mathfrak{m}^{r} \subseteq I
$$

Therefore $\tau\left(\mathfrak{a}_{\ell}^{c+\varepsilon}\right) \subseteq I$, which completes the proof of the proposition.

Let $I$ be an ideal of a ring $R$. Recall that the integral closure of $I$, denoted by $\bar{I}$, is the ideal of $R$ consisting of all $z$ that satisfy an equation $f(z)=0$ for some

$$
f(X)=X^{n}+a_{1} X^{n-1}+\cdots+a_{n} \quad\left(a_{i} \in I^{i}\right) .
$$

The ideal $I$ is integrally closed if $I=\bar{I}$. It is an immediate consequence of the definition that all multiplier ideals are integrally closed (see [Laz, Corollary 9.6.13]). 
In positive characteristic, the generalized test ideal of $\tau\left(\mathfrak{a}^{t}\right)$ is integrally closed for every $t \in \mathbb{R}_{\geq 0}$ if $\mathfrak{a}$ is generated by monomials in a polynomial ring (in fact, in this case, the test ideals are given by the same formula as the multiplier ideals in characteristic zero, see Theorem 6.10 in [HY]). More precisely, if the ideal $\mathfrak{a}$ is generated by monomials in a polynomial ring, then

$$
\tau\left(\mathfrak{a}^{t}\right)=\left\{x^{u} \in R \mid u+(1,1, \ldots, 1) \in \operatorname{Int}(t \cdot P(\mathfrak{a}))\right\}
$$

where $P(\mathfrak{a})$ is the Newton polyhedron associated to $\mathfrak{a}$.

We mention that in dimension two, Lipman and Watanabe [LW] and Favre and Jonsson [FJ] independently proved that every integrally closed ideal is the multiplier ideal of some ideal. There was some belief that such a result would be true in higher dimensions. However, recent work of Lazarsfeld and Lee [LL] shows that in fact multiplier ideals have to satisfy also some strong properties in terms of their local syzygies, allowing to give examples in dimension $\geq 3$ of integrally closed ideals that are not multiplier ideals.

However, as Theorem 3.1 clearly shows, the situation for test ideals in positive characteristic is drastically different. Since any ideal is a test ideal, in particular we get many non-integrally closed test ideals. Here is a concrete such example.

EXAMPle 3.4. Let $R=\mathbb{F}_{2} \llbracket x, y, z \rrbracket$ and $f=x^{2}+y^{5}+z^{5}$. It follows from Proposition 3.2 that $\tau\left(f^{1 / 2}\right)=\left(x, y^{2}, z^{2}\right)$, hence it is not integrally closed. In fact, we will see in Proposition 4.2 below that $f$ has no jumping numbers in $(1 / 2,1)$. It follows that we may apply Proposition 3.3 with $\varepsilon=5 / 11$ and $r=3$ to deduce that if $\mathfrak{a}=(f)+(x, y, z)^{11}$, then $\tau\left(\mathfrak{a}^{21 / 22}\right)=\left(x, y^{2}, z^{2}\right)$.

Remark 3.5. Suppose that $(R, \mathfrak{m})$ is a two-dimensional excellent Gorenstein $F$-rational local domain of characteristic $p>0$. If $\mathfrak{a} \subseteq R$ is an $\mathfrak{m}$-primary integrally closed ideal, and if $\mathfrak{b}$ is its minimal reduction, then $\tau(\mathfrak{a})=\mathfrak{b}: \mathfrak{a}$, hence $\tau(\mathfrak{a})$ is integrally closed. See [HWY, Theorem 3.1] and [HY, Theorem 5.1].

Remark 3.6. In the case of a polynomial ring we do not need the assumption that the ring is $F$-finite. More precisely, if $R=k\left[x_{1}, \ldots, x_{n}\right]$ is a polynomial ring over a field $k$ of positive characteristic, then every ideal $I$ in $R$ can be expressed as a generalized test ideal.

To see this, write $I=\left(z_{1}, \ldots, z_{\mu}\right)$, and let $k_{0}$ be the subfield of $k$ generated over the prime field $\mathbb{F}_{p}$ by the coefficients of $z_{1}, \ldots, z_{\mu}$. Since $k_{0}$ 
is an extension of finite type of a perfect field, it follows that $k_{0}$ is $F$-finite. Therefore $S=k_{0}\left[x_{1}, \ldots, x_{n}\right]$ is also $F$-finite, and we may apply Theorem 3.1 for $S$ to find $f \in S$ and $c \in \mathbb{Q}$ such that $\tau\left((f S)^{c}\right)=\left(z_{1}, \ldots, z_{\mu}\right) S$. Since $R$ is free over $S$, one can easily see that $\tau\left((f S)^{c}\right) R=\tau\left((f R)^{c}\right)$, hence $I=\tau\left((f R)^{c}\right)$.

It would be interesting to determine also in the singular case those ideals that can be written as generalized test ideals. We end this section with the following question of Shunsuke Takagi.

Question 3.7. Is the analogue of Theorem 3.1 true if we only assume that the ring is strongly $F$-regular?

\section{§4. Miscellaneous examples}

In this section we give several examples to show that the analogues of several basic properties of multiplier ideals (which follow easily from definition) fail for test ideals. We start by describing the questions we will consider.

Question 4.1. Let $(R, \mathfrak{m})$ be an $F$-finite regular local ring of characteristic $p>0$ with $d=\operatorname{dim} R \geq 1$. Let $f$ be a nonzero element of $R$, and set $c=\operatorname{fpt}(f)$. Given $t>0$, we put $\tau\left(f^{t-}\right)=\tau\left(f^{t-\varepsilon}\right)$ for $0<\varepsilon \ll 1$ (note that this is well-defined, since the $F$-jumping exponents of $f$ are discrete; see $[\mathrm{BMS1}])$.

1) Is the ideal $\tau\left(f^{c}\right)$ radical?

2) Suppose that $\tau\left(f^{c}\right)$ is $\mathfrak{m}$-primary. Is there an $\mathfrak{m}$-primary ideal $\mathfrak{b}$ such that $f \in \mathfrak{b}$ and $\operatorname{fpt}(f)=\operatorname{fpt}(\mathfrak{b})$ ?

3) Does the inclusion

$$
\mathfrak{b}^{m} \cdot \tau\left(f^{t-}\right) \cap \tau\left(f^{t}\right) \subseteq \mathfrak{b}^{m-d} \cdot \tau\left(f^{t}\right)
$$

hold for every $m \geq d$ and every $t>0$ ?

4) Does the analogue of the Generic Restriction Theorem for multiplier ideals (see Theorem 4.6 below) hold for generalized test ideals?

We recall the argument for 1) and 2) in the case of multiplier ideals. Suppose that $\mathfrak{a}$ is a nonzero ideal sheaf on the nonsingular variety $X$ (over 
an algebraically closed field of characteristic zero). Let $\pi: \widetilde{X} \rightarrow X$ be a $\log$ resolution of the pair $(X, V(\mathfrak{a}))$. If $\mathfrak{a} \mathcal{O}_{\widetilde{X}}=\mathcal{O}(-F)$, we write

$$
F=\sum_{i=1}^{r} a_{i} E_{i}, \quad K_{\tilde{X} / X}=\sum_{i=1}^{r} k_{i} E_{i} .
$$

Suppose that $c=\operatorname{lct}(\mathfrak{a})$, hence $c=\min _{i}\left(k_{i}+1\right) / a_{i}$.

The analogue of 1$)$ above holds since $\mathcal{J}\left(\mathfrak{a}^{c}\right)$ is the radical ideal corresponding to $\bigcup_{i} f\left(E_{i}\right)$, the union being over those $i$ such that $c=\left(k_{i}+1\right) / a_{i}$. Moreover, suppose that $x \in X$ is a closed point corresponding to the ideal $\mathfrak{m}$. If $\mathcal{J}\left(\mathfrak{a}^{c}\right)$ is $\mathfrak{m}$-primary, it follows that there is a divisor $E_{i}$ lying over $x$, such that $c=\left(k_{i}+1\right) / a_{i}$. In this case, for every $\ell>a_{i}$, we have $\operatorname{ord}_{E_{i}}(f)=\operatorname{ord}_{E_{i}}\left((f)+\mathfrak{m}^{\ell}\right)$. Therefore $c \geq \operatorname{lct}\left((f)+\mathfrak{m}^{\ell}\right)$, and we get the assertion in 2 ), since the reverse inequality is trivial.

The motivation for the question in 3) comes from its relevance to uniform Artin-Rees results. The corresponding statement for multiplier ideals is Theorem 3.1 in [ELSV]. The proof uses only the definition via log resolutions and Skoda's Theorem (which also holds in the setting of test ideals). It is used to give an effective uniform Artin-Rees statement for every ideal that can be written as a multiplier ideal. Therefore, in light of our Theorem 3.1, a positive answer to 3) would have had very strong consequences. It is conceivable that some weaker version of 3) might still hold, enough to give effective uniform Artin-Rees for every ideal in positive characteristic.

Our main source of counterexamples to the above questions is the following proposition, giving a formula for all the test ideals of a certain class of principal ideals.

Proposition 4.2. Let $p$ be a prime number, $n$ a positive integer, and let $R=\mathbb{F}_{p} \llbracket x_{0}, x_{1}, \ldots, x_{n} \rrbracket$ be a formal power series ring over $\mathbb{F}_{p}=\mathbb{Z} / p \mathbb{Z}$. For any nonnegative integers $\ell_{1}, \ldots, \ell_{n}$, we set

$$
f=x_{0}^{p}+x_{1}^{\ell_{1} p+1}+\cdots+x_{n}^{\ell_{n} p+1} \text { and } I=\left(x_{0}, x_{1}^{\ell_{1}}, \ldots, x_{n}^{\ell_{n}}\right) .
$$

Then

$$
\tau\left(f^{t}\right)=\left\{\begin{array}{cl}
R, & \left(0 \leq t<\frac{1}{p}\right) \\
I, & \left(\frac{1}{p} \leq t<\frac{2}{p}\right) \\
\vdots & \vdots \\
I^{p-1}, & \left(\frac{p-1}{p} \leq t<1\right) \\
f R, & (t=1) .
\end{array}\right.
$$


In particular,

(1) $\operatorname{fpt}(f)=1 / p$ and $\tau\left(f^{\mathrm{fpt}(f)}\right)=I$.

(2) For every $t \in \mathbb{R}_{\geq 0}$, we have

$$
\tau\left(f^{t}\right)=f^{\lfloor t\rfloor} I^{\lfloor p(t-\lfloor t\rfloor)\rfloor} .
$$

(3) The set of F-jumping exponents of $f$ is $\frac{1}{p} \mathbb{Z}_{\geq 0}$.

Proof. It is enough to show that $\tau\left(f^{t}\right)=I^{r}$ for $t \in\left[\frac{r}{p}, \frac{r+1}{p}\right)$ and for every $r=0,1, \ldots, p-1$. The other assertions follow from this and Skoda's Theorem. First, we show the following

Claim 1. $\tau\left(f^{r / p}\right)=I^{r}$.

Since we have

$$
\begin{aligned}
f^{\left\lceil(r / p) p^{e}\right\rceil} & =f^{r p^{e-1}}=\left(x_{0}^{p^{e}}+x_{1}^{\ell_{1} p^{e}+p^{e-1}}+\cdots+x_{n}^{\ell_{n} p^{e}+p^{e-1}}\right)^{r} \\
& =\sum_{\substack{i_{0}, \ldots, i_{n} \geq 0 \\
i_{0}+\cdots+i_{n}=r}} \frac{r !}{i_{0} ! i_{1} ! \cdots i_{n} !}\left(x_{0}^{i_{0}} x_{1}^{\ell_{1} i_{1}} \cdots x_{n}^{\ell_{n} i_{n}}\right)^{p^{e}} x_{1}^{i_{1} p^{e-1}} \cdots x_{n}^{i_{n} p^{e-1}}
\end{aligned}
$$

and since $\left\{\frac{r !}{i_{0} ! i_{1} ! \cdots i_{n} !} x_{1}^{i_{1} p^{e-1}} \cdots x_{n}^{i_{n} p^{e-1}}\right\}$ is part of a free basis of $R$ over $R^{p^{e}}$, we obtain that

$$
\left(f^{\left\lceil(r / p) p^{e}\right\rceil}\right)^{\left[1 / p^{e}\right]}=\left(x_{0}, x_{1}^{\ell_{1}}, \ldots, x_{n}^{\ell_{n}}\right)^{r} .
$$

Since this holds for every $e \geq 1$, we get our claim.

In order to prove that $\tau\left(f^{t}\right)=I^{r}$ when $\frac{r}{p}<t<\frac{r+1}{p}$, we put $t=$ $\frac{r+1}{p}-\varepsilon, 0<\varepsilon<\frac{1}{p}$. It follows from Claim 1 that it is enough to show that $I^{r} \subseteq \tau\left(f^{t}\right)$. We fix a sufficiently large integer $e$ such that $s:=\left\lfloor\varepsilon p^{e}\right\rfloor \geq 1$. We have

$$
\begin{aligned}
f^{\left\lceil t p^{e}\right\rceil} & =\left(x_{0}^{p}+x_{1}^{\ell_{1} p+1}+\cdots+x_{n}^{\ell_{n} p+1}\right)^{(r+1) p^{e-1}-s} \\
& =\sum_{\substack{a_{0}, \ldots, a_{n} \geq 0 \\
a_{0}+\cdots+a_{n}=(r+1) p^{e-1}-s}} \frac{\left((r+1) p^{e-1}-s\right) !}{a_{0} ! a_{1} ! \cdots a_{n} !} x_{0}^{p a_{0}} x_{1}^{\left(\ell_{1} p+1\right) a_{1}} \cdots x_{n}^{\left(\ell_{n} p+1\right) a_{n}} .
\end{aligned}
$$


In order to complete the proof, it is enough to show that for every $(n+1)$ tuple of nonnegative integers $\left(i_{0}, i_{1}, \ldots, i_{n}\right)$ such that $i_{0}+i_{1}+\cdots+i_{n}=r$, we have

$$
y:=x_{0}^{i_{0}} x_{1}^{\ell_{1} i_{1}} \cdots x_{n}^{\ell_{n} i_{n}} \in\left(f^{\left\lceil t p^{e}\right\rceil}\right)^{\left[1 / p^{e}\right]} .
$$

If we put $a_{0}=\left(i_{0}+1\right) p^{e-1}-s, a_{j}=i_{j} p^{e-1}$ for $j=1, \ldots, n$, then we have

$$
a_{0}, a_{1}, \ldots, a_{n} \geq 0, \quad a_{0}+a_{1}+\cdots+a_{n}=(r+1) p^{e-1}-s
$$

and

$$
x_{0}^{p a_{0}} x_{1}^{\left(\ell_{1} p+1\right) a_{1}} \cdots x_{n}^{\left(\ell_{n} p+1\right) a_{n}}=\left(x_{0}^{i_{0}} x_{1}^{\ell_{1} i_{1}} \cdots x_{n}^{\ell_{n} i_{n}}\right)^{p^{e}} x_{0}^{p^{e}-s p} x_{1}^{i_{1} p^{e-1}} \cdots x_{n}^{i_{n} p^{e-1}} .
$$

Therefore it is enough to prove the claim below. Note that the claim implies that $f^{\left\lceil t p^{e}\right\rceil}$ can be written as $y_{1}^{p^{e}} g_{1}+\cdots+y_{\mu}^{p^{e}} g_{\mu}$, such that $I^{r}=\left(y_{1}, \ldots, y_{\mu}\right)$ and $\left\{g_{1}, \ldots, g_{\mu}\right\}$ is part of a free basis of $R$ over $R^{p^{e}}$.

\section{Claim 2.}

(1) $\frac{\left((r+1) p^{e-1}-s\right) !}{a_{0} ! a_{1} ! \cdots a_{n} !} \not \equiv 0(\bmod p)$.

(2) Let $b_{0}, b_{1}, \ldots, b_{n} \geq 0$ be integers with $b_{0}+b_{1}+\cdots+b_{n}=(r+1) p^{e-1}-s$. If there exist $t_{0}, t_{1}, \ldots, t_{n} \in \mathbb{Z}$ such that

$$
p b_{0}-p a_{0}=t_{0} p^{e}, \quad\left(\ell_{j} p+1\right)\left(b_{j}-a_{j}\right)=t_{j} p^{e}(j=1, \ldots, n),
$$

then $b_{0}=a_{0}, b_{1}=a_{1}, \ldots, b_{n}=a_{n}$.

In order to prove (1), we use the fact that for every integer $N$, the order of $p$ in $N$ ! is $\sum_{m \geq 1}\left\lfloor N / p^{m}\right\rfloor$. Note that if $1 \leq m \leq e-1$, then we have

$$
\left\lfloor\left(a_{0}+a_{1}+\ldots a_{n}\right) / p^{m}\right\rfloor=\left\lfloor a_{0} / p^{m}\right\rfloor+\sum_{j=1}^{n} i_{j} p^{e-1-m}=\sum_{j=0}^{n}\left\lfloor a_{j} / p^{m}\right\rfloor .
$$

On the other hand, $a_{0}+a_{1}+\cdots+a_{n}<p^{e}$. This shows that the order of $p$ in $\frac{\left((r+1) p^{e-1}-s\right) !}{a_{0} ! a_{1} ! \cdots a_{n} !}$ is zero.

We now prove $(2)$. Since $\operatorname{gcd}\left(p, \ell_{j} p+1\right)=1$, we have $p^{e} \mid\left(b_{j}-a_{j}\right)$ for every $1 \leq j \leq n$. Therefore we can write $b_{j}-a_{j}=u_{j} p^{e}$ for every $j$ as above, and suitable $u_{j} \in \mathbb{Z}$. Using $b_{j}=\left(i_{j}+p u_{j}\right) p^{e-1} \geq 0$, we deduce $i_{j}+p u_{j} \geq 0$, hence $u_{j} \geq 0$ (recall that $i_{0}+\cdots+i_{n}=r<p$ ). On the other hand, since $b_{0}=\left(i_{0}+1+t_{0}\right) p^{e-1}-s \geq 0$, we get $i_{0}+1+t_{0}>0$ and thus $t_{0} \geq-i_{0}>-p$. Moreover, $a_{0}+\cdots+a_{n}=b_{0}+\cdots+b_{n}$ yields $\left(u_{1}+\cdots+u_{n}\right) p+t_{0}=0$. Therefore $a_{j}=b_{j}$ for every all $j$. This completes the proof of Claim 2, and also the proof of the proposition. 
EXAmple 4.3. Let $R=\mathbb{F}_{2} \llbracket x, y, z \rrbracket, f=x^{2}+y^{5}+z^{5}$, and put $\mathfrak{a}_{N}=$ $(f)+(x, y, z)^{N}$ for every $N \geq 1$.

(1) $\operatorname{fpt}(f)=1 / 2$.

(2) $\tau\left(f^{\mathrm{fpt}(f)}\right)=\left(x, y^{2}, z^{2}\right)$ is an $\mathfrak{m}$-primary ideal, but it is not radical (hence this gives a counterexample to 1) in Question 4.1.

(3) $\operatorname{fpt}\left(\mathfrak{a}_{N}\right)>\operatorname{fpt}(f)=1 / 2$ for every $N \geq 1$ (hence this gives a counterexample to 2) in Question 4.1).

Proof. (1) and (2) follow from Proposition 4.2. In order to see that (3) indeed says that we get a counterexample to 2) in Question 4.1, note that if $\mathfrak{b}$ is an $\mathfrak{m}$-primary ideal containing $f$, then there is $N \geq 1$ such that $\mathfrak{a}_{N} \subseteq \mathfrak{b}$. Hence $\operatorname{fpt}(\mathfrak{b}) \geq \operatorname{fpt}\left(\mathfrak{a}_{N}\right)>\operatorname{fpt}(f)$.

It is enough to prove the assertion in (3) for every $N=2^{e-2}$, where $e \geq 5$. We show that in this case $\mathfrak{a}_{N}^{2^{e-1}} \nsubseteq\left(x^{2^{e}}, y^{2^{e}}, z^{2^{e}}\right)$, hence $\tau\left(\mathfrak{a}_{N}^{1 / 2}\right)=R$. Consider

$$
h:=f^{2^{e-1}-4} x^{N} y^{N} z^{2 N} \in \mathfrak{a}_{N}^{2^{e-1}} .
$$

If $a=2\left(2^{e-1}-4-2^{e-3}\right)+2^{e-2}=2^{e}-8, b=5 \cdot 2^{e-3}+2^{e-2}=7 \cdot 2^{e-3}$, and $c=2^{e-1}$, then the monomial $x^{a} y^{b} z^{c}$ is not in $\left(x^{2^{e}}, y^{2^{e}}, z^{2^{e}}\right)$, and its coefficient in $h$ is $\left(\begin{array}{c}2^{e-1}-4 \\ 2^{e-3}\end{array}\right)$. In order to show that this coefficient is nonzero, we compute the order of 2 in $\left(\begin{array}{c}2^{e-1}-4 \\ 2^{e-3}\end{array}\right)$. This order is equal to

$$
\begin{aligned}
& \sum_{i=1}^{e-2}\left(\left\lfloor\left(2^{e-1}-4\right) / 2^{i}\right\rfloor-\left\lfloor 2^{e-3} / 2^{i}\right\rfloor-\left\lfloor\left(2^{e-1}-4-2^{e-3}\right) / 2^{i}\right\rfloor\right) \\
& \quad=\left\lfloor\left(2^{e-1}-4\right) / 2^{e-2}\right\rfloor-\left\lfloor\left(2^{e-1}-4-2^{e-3}\right) / 2^{e-2}\right\rfloor=1-1=0 .
\end{aligned}
$$

This concludes the proof of (3).

Remark 4.4. Karl Schwede [Sch] has recently introduced the notion of sharp F-purity. He proved that if $c=\operatorname{fpt}(f)<1$ is such that the denominator of $c$ is not divisible by $p$, then the ideal $\tau\left(f^{c}\right)$ is radical; see Corollary 4.3 and Remark 5.5 in loc. cit. It would be very interesting to see whether assuming that the denominators of the jumping numbers of $f$ are not divisible by $p$ would imply other good properties of the generalized test ideals of $f$.

We consider now the third problem in Question 4.1. 
EXAMPLE 4.5. Let $p$ be a prime, $R=\mathbb{F}_{p} \llbracket x, y \rrbracket$ and $f=x^{p}+y^{\ell p+1}$, for some $\ell \geq 3$. It follows from Proposition 4.2 that $\operatorname{fpt}(f)=1 / p$ and $\tau\left(f^{1 / p}\right)=\left(x, y^{\ell}\right)$. If we take $\mathfrak{b}=(y)$ and $t=1 / p$, then we see that

$$
\mathfrak{b}^{\ell} \cdot \tau\left(f^{t-}\right) \cap \tau\left(f^{t}\right)=\mathfrak{b}^{\ell} \cap\left(x, y^{\ell}\right)=\left(y^{\ell}\right) \nsubseteq \mathfrak{b}^{\ell-2} \cdot \tau\left(f^{t}\right)=\left(y^{\ell-2}\right) \cdot\left(x, y^{\ell}\right),
$$

giving thus a counterexample to 3) in Question 4.1.

We conclude this section with a discussion of the analogue of the Generic Restriction Theorem for multiplier ideals in the characteristic $p$ setting. Let us recall the result in characteristic zero (see [Laz, Theorem 9.5.35 and Example 9.5.37]).

THEOREM 4.6. Let $f: X \rightarrow S$ be a smooth surjective morphism of nonsingular complex algebraic varieties. If $\mathfrak{a}$ is a sheaf of ideals on $X$, then there is an open subset $U \subseteq S$ such that

$$
\mathcal{J}\left(X, \mathfrak{a}^{c}\right) \cdot \mathcal{O}_{X_{s}}=\mathcal{J}\left(X_{s},\left(\mathfrak{a} \cdot \mathcal{O}_{X_{s}}\right)^{c}\right)
$$

for every $s \in U$ and every positive $c$ (here $X_{s}$ denotes the fiber $f^{-1}(s)$ ).

We show now that the analogue of this result fails for test ideals. Suppose, for simplicity, that $k$ is an algebraically closed field of positive characteristic, and consider $f \in R=k\left[x_{1}, \ldots, x_{n}, y\right]$. Let us denote by $\left\{u_{j}\right\}_{j}$ the monomials $x_{1}^{a_{1}} \cdots x_{n}^{a_{n}}$, where $0 \leq a_{i} \leq p-1$ for every $i$. We write

$$
f=\sum_{i=0}^{p-1} y^{i} \sum_{j} u_{j} g_{i j}(x, y)^{p}
$$

for some $g_{i j} \in R$. Arguing as in the proof of Proposition 3.2, we see that

$$
\tau\left(f^{1 / p}\right)=(f)^{[1 / p]}=\left(g_{i j}(x, y) \mid i, j\right)
$$

On the other hand, let us put $f_{\lambda}(x):=f(x, \lambda) \in k\left[x_{1}, \ldots, x_{n}\right]$ for every $\lambda \in k$. Note that we have

$$
f_{\lambda}=\sum_{j} u_{j} \sum_{i=0}^{p-1} g_{i j}(x, \lambda)^{p} \lambda^{i},
$$

hence we deduce

$$
\tau\left(f_{\lambda}^{1 / p}\right)=\left(f_{\lambda}\right)^{[1 / p]}=\left(\sum_{i=0}^{p-1} \lambda^{i / p} g_{i j}(x, \lambda) \mid j\right) .
$$


EXAmple 4.7. Consider $f \in k\left[x_{1}, x_{2}, y\right]$ given by $f\left(x_{1}, x_{2}, y\right)=x_{1}^{p}+$ $x_{2}^{p} y$. The above discussion implies that $\tau\left(f^{1 / p}\right)=\left(x_{1}, x_{2}\right)$, while for every $\lambda \in k$ we have $\tau\left(f_{\lambda}^{1 / p}\right)=\left(x_{1}+\lambda^{1 / p} x_{2}\right)$. This gives a negative answer to 4$)$ in Question 4.1.

The main application of Theorem 4.6 is to prove the semicontinuity of $\log$ canonical thresholds. In spite of the above example, we will see in the next section that the analogous result for $F$-pure thresholds holds.

\section{§5. Semicontinuity of $\boldsymbol{F}$-pure thresholds}

The following theorem is the analogue of the Semicontinuity Theorem for log canonical thresholds (see [Laz, Example 9.5.41]).

TheOREM 5.1. Let $f: R \rightarrow S$ be an algebra homomorphism between two $k$-algebras of finite type, where $k$ is a field of characteristic $p$, with $\left[k: k^{p}\right]<\infty$. We assume that all fibers of $f$ are nonsingular, of pure dimension d. Let $\phi: S \rightarrow R$ be a ring homomorphism such that $\phi \circ f=\operatorname{id}_{R}$, and for every $\mathfrak{q} \in \operatorname{Spec}(R)$, we put $\mathfrak{q}^{\prime}=\phi^{-1}(\mathfrak{q})$. For every ideal $\mathfrak{a}$ in $S$ such that $\mathfrak{a} \subseteq \mathfrak{q}^{\prime}$ for all $\mathfrak{q} \in \operatorname{Spec}(R)$, and for every nonnegative $c$, the set

$$
\left\{\mathfrak{q} \in \operatorname{Spec}(R) \mid \operatorname{fpt}\left(\mathfrak{a} \cdot S_{\mathfrak{q}^{\prime}} / q S_{\mathfrak{q}^{\prime}}\right) \geq c\right\}
$$

is open in $\operatorname{Spec}(R)$.

Proof. Note that for every $\mathfrak{q} \in \operatorname{Spec}(R)$ we have $\left[k(\mathfrak{q}): k(\mathfrak{q})^{p}\right]<\infty$, hence the ring $S_{\mathfrak{q}^{\prime}} / \mathfrak{q} S_{\mathfrak{q}^{\prime}}$ is $F$-finite and regular. Consider a surjective morphism of $R$-algebras $g: T=R\left[x_{1}, \ldots, x_{n}\right] \rightarrow S$. We claim that we may replace $S$ by $R\left[x_{1}, \ldots, x_{n}\right]$. Indeed, it follows from Proposition 3.6 in [BMS1] that if we write $\mathfrak{a}=\mathfrak{b} / \operatorname{ker}(g)$ and $\mathfrak{q}^{\prime}=\mathfrak{q}^{\prime \prime} / \operatorname{ker}(g)$, then

$$
\operatorname{fpt}\left(\mathfrak{a} \cdot S_{\mathfrak{q}^{\prime}} / \mathfrak{q} S_{\mathfrak{q}^{\prime}}\right)+n-d=\operatorname{fpt}\left(\mathfrak{b} \cdot T_{\mathfrak{q}^{\prime \prime}} / \mathfrak{q} T_{\mathfrak{q}^{\prime \prime}}\right) .
$$

This proves our claim. Moreover, note that if $\phi: S=R\left[x_{1}, \ldots, x_{n}\right] \rightarrow R$ is given by $\phi\left(x_{i}\right)=b_{i}$, then we may consider the automorphism of $R$-algebras $\rho: S \rightarrow S$ given by $\rho\left(x_{i}\right)=x_{i}+b_{i}$. After replacing $\mathfrak{a}$ by $\rho(\mathfrak{a})$, we may also assume that $\phi\left(x_{i}\right)=0$ for every $i$. We see that for every $\mathfrak{q} \in \operatorname{Spec}(R)$, we are interested in the $F$-pure threshold of $\mathfrak{a} \cdot k(\mathfrak{q})\left[x_{1}, \ldots, x_{n}\right]_{\left(x_{1}, \ldots, x_{n}\right)}$, that we denote by $\operatorname{fpt}_{0}\left(\mathfrak{a} \cdot k(\mathfrak{q})\left[x_{1}, \ldots, x_{n}\right]\right)$.

Let us choose generators $g_{1}, \ldots, g_{m}$ for $\mathfrak{a}$, and let $D=\max _{i}\left\{\operatorname{deg}\left(g_{i}\right)\right\}$. It follows from Proposition 3.8 in [BMS1] that there is $N=N(D, n, m)$ such 
that the denominator of every $F$-jumping exponent of an ideal of the form $\mathfrak{a}$. $k(\mathfrak{q})\left[x_{1}, \ldots, x_{n}\right]($ for $\mathfrak{q} \in \operatorname{Spec}(R))$ is $\leq N$. Note that $\operatorname{fpt}_{0}\left(\mathfrak{a} \cdot k(\mathfrak{q})\left[x_{1}, \ldots, x_{n}\right]\right)$ is an $F$-jumping exponent of $\mathfrak{a} \cdot k(\mathfrak{q})\left[x_{1}, \ldots, x_{n}\right]$ (though it might be larger than the $F$-pure threshold of this ideal). Using also the fact that the $F$-pure threshold of an ideal in a regular ring of dimension $n$ is $\leq n$, we deduce that the set

$$
\left\{\operatorname{fpt}_{0}\left(\mathfrak{a} \cdot k(\mathfrak{q})\left[x_{1}, \ldots, x_{n}\right]\right) \mid \mathfrak{q} \in \operatorname{Spec}(R)\right\}
$$

is finite.

In particular, in order to prove the theorem, we may choose the largest element $c^{\prime}$ in the above set, with $c^{\prime}<c$. It is enough to show that the set

$$
A_{c^{\prime}}:=\left\{\mathfrak{q} \in \operatorname{Spec}(R) \mid \operatorname{fpt}_{0}\left(\mathfrak{a} \cdot k(\mathfrak{q})\left[x_{1}, \ldots, x_{n}\right]\right) \leq c^{\prime}\right\}
$$

is closed. Using the description of the $F$-pure threshold in (3) in Section 2, we see that $A_{c^{\prime}}=\bigcap_{e \geq 1} A_{c^{\prime}, e}$, where

$$
A_{c^{\prime}, e}=\left\{\mathfrak{q} \mid \mathfrak{a}^{\left\lfloor c^{\prime} p^{e}\right\rfloor+1} \subseteq\left(x_{1}^{p^{e}}, \ldots, x_{n}^{p^{e}}\right) \text { in } k(\mathfrak{q})\left[x_{1}, \ldots, x_{n}\right]\right\}
$$

Note that if we consider all $g^{\ell}:=g_{1}^{\ell_{1}} \cdots g_{m}^{\ell_{m}}$, with $\sum_{i} \ell_{i}=\left\lfloor c^{\prime} p^{e}\right\rfloor+1$, then $A_{c^{\prime}, e}$ is the set of primes $\mathfrak{q}$ containing all the coefficients of monomials not in $\left(x_{1}^{p^{e}}, \ldots, x_{n}^{p^{e}}\right)$, in all $g^{\ell}$ as above. Therefore each $A_{c^{\prime}, e}$ is a closed subset of $\operatorname{Spec}(R)$, hence $A_{c^{\prime}}$ is closed, too.

Acknowledgements. We are indebted to Shunsuke Takagi and to Kei-ichi Watanabe for inspiring discussions.

\section{REFERENCES}

[BMS1] M. Blickle, M. Mustaţă and K. E. Smith, Discreteness and rationality of Fthresholds, Michigan Math. J., 57 (2008), 43-61.

[BMS2] M. Blickle, M. Mustaţă and K. E. Smith, F-thresholds of hypersurfaces, Trans. Amer. Math. Soc., to appear.

[ELSV] L. Ein, R. Lazarsfeld, K. E. Smith, and D. Varolin, Jumping coefficients of multiplier ideals, Duke Math. J., 123 (2004), 469-506.

[FJ] C. Favre and M. Jonsson, Valuations and multiplier ideals, J. Amer. Math. Soc., 18 (2005), 655-684.

[Ha] N. Hara, with an appendix by P. Monsky, F-pure thresholds and F-jumping coefficients in dimension two, Math. Res. Lett., 13 (2006), 747-760.

[HT] N. Hara and S. Takagi, On a generalization of test ideals, Nagoya Math. J., 175 (2004), 59-74. 
[HY] N. Hara and K. Yoshida, A generalization of tight closure and multiplier ideals, Trans. Amer. Math. Soc., 355 (2003), 3143-3174.

[HWY] N. Hara, K.-i. Watanabe and K. Yoshida, F-rationality of Rees algebras, J. Algebra, 247 (2002), 153-190.

$[\mathrm{Ku}]$ E. Kunz, Characterization of regular local rings in characteristic $p$, Amer. J. Math., 91 (1969), 772-784.

[Laz] R. Lazarsfeld, Positivity in Algebraic Geometry II, Ergebnisse der Mathematik und ihrer Grenzgebiete. 3. Folge, A Series of Modern Surveys in Mathematics, Vol. 49, Springer-Verlag, Berlin, 2004.

[LL] R. Lazarsfeld and K. Lee, Local syzygies of multiplier ideals, Invent. Math., 167 (2007), 409-418.

[LW] J. Lipman and K.-i. Watanabe, Integrally closed ideals in two-dimensional regular local rings are multiplier ideals, Math. Res. Letters, 10 (2003), 423-434.

[MTW] M. Mustaţă, S. Takagi and K.-i. Watanabe, F-thresholds and Bernstein-Sato polynomials, European Congress of Mathematics, Eur. Math. Soc., Zürich, 2005, pp. 341-364.

[Sch] K. Schwede, Generalized test ideals, sharp F-purity, and sharp test elements, Math. Res. Lett., 15 (2008), 1251-1262.

[Ta] S. Takagi, Formulas for multiplier ideals on singular varieties, Amer. J. Math., 128 (2006), 1345-1362.

[TW] S. Takagi and K.-i. Watanabe, On F-pure thresholds, J. Algebra, 282 (2004), $278-297$.

Mircea Mustaţă

Department of Mathematics

University of Michigan

Ann Arbor, MI 48109

$U S A$

mmustata@umich.edu

Ken-ichi Yoshida

Graduate School of Mathematics

Nagoya University

Chikusa-ku

Nagoya, 464-8602

Japan

yoshida@math.nagoya-u.ac.jp 